Case Report

\title{
Canine Oral Eosinophilic Granuloma Treated with Electrochemotherapy
}

\author{
Matías Nicolás Tellado, ${ }^{1}$ Sebastián Diego Michinski, ${ }^{2,3}$ Nahuel Olaiz, ${ }^{3}$ \\ Felipe Maglietti, ${ }^{3}$ and Guillermo Marshall ${ }^{3}$ \\ ${ }^{1}$ Facultad de Ciencias Veterinarias, Universidad de Buenos Aires, Avenida Chorroarín 280, \\ C1427CWO Ciudad de Buenos Aires, Argentina \\ ${ }^{2}$ Instituto Tecnológico de Buenos Aires, Avenida Eduardo Madero 399, C1106ACD Ciudad de Buenos Aires, Argentina \\ ${ }^{3}$ Laboratorio de Sistemas Complejos, Facultad de Ciencias Exactas y Naturales, Universidad de Buenos Aires, \\ Intendente Guiraldes 2160, Pabellon I, C1428EGA Ciudad de Buenos Aires, Argentina
}

Correspondence should be addressed to Guillermo Marshall; marshallg@arnet.com.ar

Received 24 June 2014; Accepted 7 October 2014; Published 16 October 2014

Academic Editor: Isabel Pires

Copyright (C) 2014 Matías Nicolás Tellado et al. This is an open access article distributed under the Creative Commons Attribution License, which permits unrestricted use, distribution, and reproduction in any medium, provided the original work is properly cited.

\begin{abstract}
A case of a canine oral eosinophilic granuloma in a 14-year-old female crossbred is described. The dog was presented with a history of ptyalism, halitosis, local pain, decreased appetite, and blood staining noted on food and water bowls. Clinical, hematologic, and biochemical examinations, abdominal ultrasonography, and 3-view chest radiographs were performed, and no metastases were found. Histopathologic examination of two $6 \mathrm{~mm}$ punch biopsies from the oral lesion revealed the presence of eosinophilic granulomatous lesions in the submucosa. After treatment with corticosteroids and wide spectrum antibiotics no significant changes in clinical signs and lesion size were observed. Electrochemotherapy (ECT), a novel tumor treatment routinely used for cutaneous and subcutaneous tumors in human patients in the European Union since 2006, was used to treat the eosinophilic granuloma. The procedure was performed under general anesthesia, followed by intravenous administration of bleomycin. Six weeks after treatment a complete response with disappearance of the mass and improvement of clinical signs were observed.
\end{abstract}

\section{Introduction}

Canine eosinophilic granuloma is an eosinophilic disease reported as a rare lesion characterized by nodules and plaques in the mouth, tonsils, or skin. The exact cause is unknown, but infectious agents as bacteria, parasites, or fungal organisms have been implicated in the pathogenesis. Siberian Huskies and Cavalier King Charles Spaniels are most commonly affected $[1,2]$. Few studies have documented the disease in older crossbred dogs. Oral lesions are characterized by plaques or proliferative masses. These are most commonly found on the palate and the lateral or ventral side of the tongue. Oral lesions may be painful and halitosis is usually the presenting complaint.

The etiology of these lesions remains unknown, although an underlying allergic condition is likely. This disease occurs in dogs as a collagen tissue degeneration [1, 3-5]. In humans, some reports have proposed several mechanisms of pathogenesis as vasculitis, microangiopathies, fibromatosis, phagocytic dysfunction, and cell mediated immune-response dysfunction [6].

Standard treatments are made with the aim of reducing the size of the granuloma and to improve the general condition of the patient. A treatment with antibiotic and corticosteroid therapy has been reported with a partial response and occurrence of side effects such as gastritis, enteritis, and chronic renal failure due to prolonged use of high doses of corticosteroids in older patients [3]. The surgical excision followed by immunosuppressive therapy with corticosteroids or azathioprine might achieve best local results, but in patients with large masses aggressive procedure is necessary. Also, a 
high rate of recurrence was observed after a short treatment period $[2,3,7]$.

ECT is a novel treatment modality consisting in the use of an electric field to increase tissue permeability to certain drugs $[8,9]$. Bleomycin is usually the drug of choice, which works as an endonuclease, cutting the DNA strands and thus interfering with cell division. This provides a sort of selectivity towards dividing cells, sparing the quiescent ones and thus allowing the tissue to heal with minimum scaring. It has been used in the European Union since 2006, for the treatment of cutaneous and subcutaneous tumors [10, 11], particularly for the treatment of melanoma nodules. Cemazar et al. reviewed the use of electrochemotherapy for treating tumors in veterinary oncology [12].

In this case report we show for the first time the use of electrochemotherapy for the treatment of a canine eosinophilic granuloma, a nononcological disease.

\section{Case Presentation}

A case of a 14-year-old canine female crossbred is described. The dog was presented with a history of ptyalism, halitosis, mild weight loss, local pain, decreased appetite, and blood staining noted on food and water bowls.

2.1. Clinical Examination. The patient's general condition was good on the physical exam; hypersalivation, halitosis, and uncomfortable chewing were the main clinical signs. A $3.8 \times 2.2 \times 1.2 \mathrm{~cm}$ bloody mass was found on the lower right side of the mouth (Figure 1). We also detected a right submandibular lymph node enlargement; thus a fine needle aspiration was performed. Complete laboratory analysis, abdominal ultrasound, and 3-view thoracic radiographs were performed as a first clinical staging. The blood count revealed mild anemia (29\% hematocrit, reference range 35-55\%), mild leukocytosis $\left(36 \times 10^{3}\right.$ leukocytes $/ \mu \mathrm{L}$, reference range $6.0-12.0 \times 10^{3}$ leukocytes $\left./ \mu \mathrm{L}\right)$, an increased percentage of segmented neutrophilic granulocytes $(83 \%$, reference range 60-75\%), 13\% lymphocytes (reference range 15-30\%), 4\% eosinophils (reference range $0-6 \%$ ), and slight thrombocytopenia $\left(250 \times 10^{3}\right.$ platelets $/ \mu \mathrm{L}$, reference range $300-500 \times$ $10^{3}$ platelets $\left./ \mu \mathrm{L}\right)$. The routine biochemical parameters were within the normal range. The cytology from the fine needle aspiration showed a reactive lymph node, likely due to the oral inflammation and infection. No metastatic signs were observed on the imaging studies; therefore two samples of $6 \mathrm{~mm}$ punch biopsy were taken from the mass to be referred to the pathologist.

Two formalin fixed samples were embedded in paraffin wax and then stained with hematoxylin and eosin (HE). The histopathology samples revealed a chronic eosinophilic granuloma consisting of densely eosinophilic material representing remnants of fragmented collagen bundles and degenerate eosinophils which have released their granules to the interstitium. At the periphery of the granuloma, mainly differentiated fibrovascular tissue can be seen with some eosinophilic granulocytes intermingled with small numbers

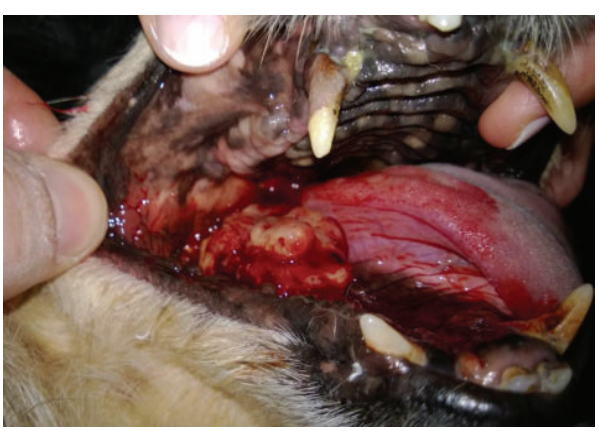

FIGURE 1: Picture taken on day 1 , before the ECT treatment. On the right side of the mouth a $3.8 \times 2.2 \times 1.2 \mathrm{~cm}$ bloody mass can be seen corresponding to an eosinophilic granuloma.

of lymphocytes, macrophages, plasma cells, and neutrophils (Figure 2).

2.2. Treatment. After a treatment with prednisolone $2 \mathrm{mg} / \mathrm{kg} / \mathrm{sid}$ and amoxicillin/clavulanic acid $62.5 \mathrm{mg} / \mathrm{kg} / \mathrm{bid}$, no significant changes in clinical signs and lesion size were observed.

A wide-margin surgical excision was indicated to ensure that all eosinophilic granuloma is removed, but this indication was not accepted by pet owners. For this reason, ECT was the therapeutic modality chosen and an informed consent was signed. Local regulations were followed (Argentina, law $\left.\mathrm{N}^{\circ} 14,072\right)$ [13].

The treatment was performed under general anesthesia. The patient was induced with intravenous bolus of propofol $(3 \mathrm{mg} / \mathrm{kg})$ and diazepam $(0.5 \mathrm{mg} / \mathrm{kg})$ and maintained after tracheal intubation with isoflurane and intravenous fentanyl $(2 \mu \mathrm{g} / \mathrm{kg})$. This scheme of anesthesia guarantees an adequate comfort of the patient during the treatment. Prophylactic antibiotics were administered.

A single dose of bleomycin $\left(15,000 \mathrm{IU} / \mathrm{m}^{2}\right)$ was administered in bolus, and the electric pulses were delivered 8 minutes later, applying 8 pulses of $800 \mathrm{~V} / \mathrm{cm}$ and $100 \mu \mathrm{sec}$ long at a repetition frequency of $10 \mathrm{~Hz}$, covering the whole lesion surface, using a pulse generator BTX ECM 830, Harvard Apparatus, Boston, USA. We used a 6-needle electrode (Figure 3), as the area covered with the electrode is $0.8 \times$ $0.8 \mathrm{~cm} ; 15$ applications were needed to cover the lesion and superposition was avoided. After the procedure, oral meloxicam $(0.1 \mathrm{mg} / \mathrm{kg} / \mathrm{sid})$ was administered for pain and local swelling control.

To determine the dose of bleomycin the body surface of the dog was estimated using the following formula [14]:

$$
\text { body surface in } \mathrm{m}^{2}=\frac{10.1 \times(\text { weight in grams })^{2 / 3}}{10000} \text {. }
$$

The weight was $8.9 \mathrm{~kg}$; thus the calculated body surface was $0.434 \mathrm{~m}^{2}$.

2.3. Followup. Four days after ECT, the patient showed mild inflammation of the gingiva, but no significant changes in food and water intake were observed by the pet owner. 

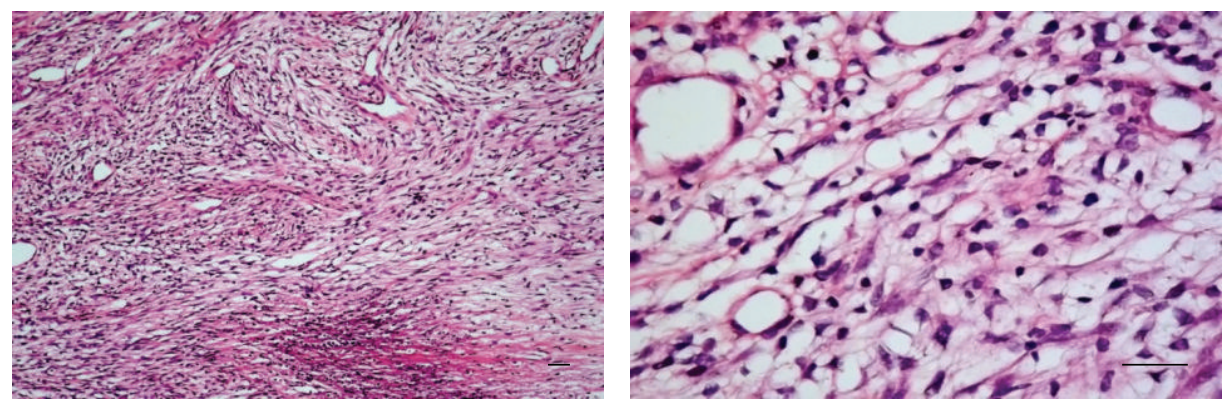

FIGURE 2: Histopathology samples stained with hematoxylin and eosin revealed a chronic eosinophilic granuloma consisting of densely eosinophilic material representing remnants of fragmented collagen bundles and degenerate eosinophils which have released their granules to the interstitium. At the periphery of the granuloma, mainly differentiated fibrovascular tissue can be seen with some eosinophilic granulocytes intermingled with small numbers of lymphocytes, macrophages, plasma cells, and neutrophils (scale bars $=100 \mu \mathrm{m})$.

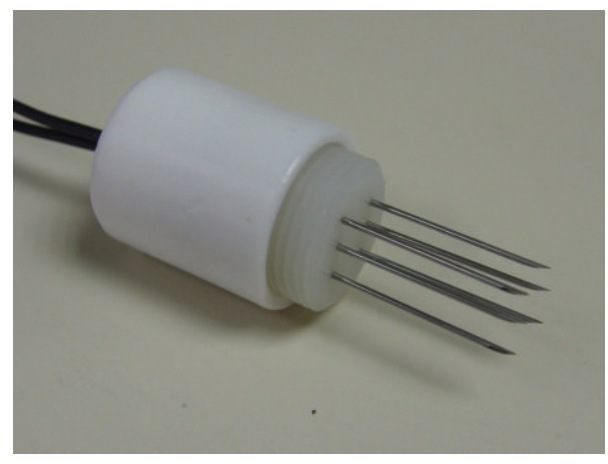

FIGURE 3: Six-needle electrode used to treat the patient. It consists of two rows of 3 needles. The separation of the rows is $0.8 \mathrm{~cm}$; the separation between the needles in the same row is $0.4 \mathrm{~cm}$. Each needle is $2 \mathrm{~cm}$ long and $1.2 \mathrm{~mm}$ in diameter.

14 days after ECT, no signs of inflammation or a clear improvement in general condition due to the partial reduction of the mass were observed. Meloxicam was discontinued due to lack of inflammation.

45 days after treatment (Figure 4) improvement of life quality was observed to be likely related to absence of pain; also decreased sialorrhea and halitosis were obvious. In accordance with the World Health Organization response criteria [15], a complete remission with a $100 \%$ reduction of the lesion size was observed.

After day 45, one month of corticosteroid therapy with prednisolone $1.5 \mathrm{mg} / \mathrm{kg} / \mathrm{sid}$ and omeprazole $1 \mathrm{mg} / \mathrm{kg} / \mathrm{sid}$ was indicated to prevent local recurrence.

\section{Conclusions and Discussion}

The standard treatment for the eosinophilic granuloma has a very low efficacy and most of its side effects may seriously affect the quality of life of the patient. We conclude that, in this case of canine oral eosinophilic granuloma, ECT was a very good treatment option with excellent results. This result confirms that one of the most remarkable aspects of ECT is its effectiveness against any histological type of tumor $[12,16,17]$. As ECT kills proliferating cells mainly by apoptosis, quiescent

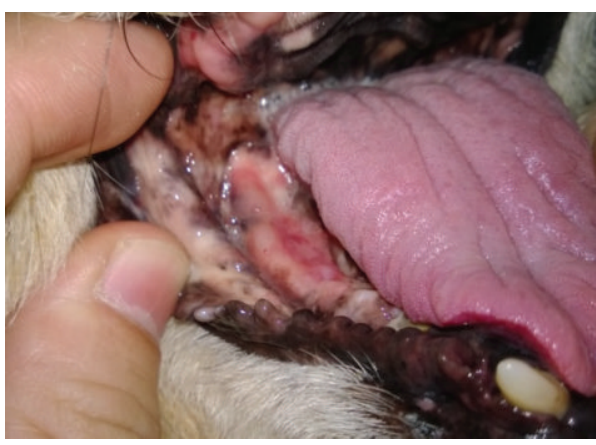

FIgURE 4: Picture taken 45 days after the ECT treatment. A complete response was achieved.

cells are preserved. In this case, ECT was a logical treatment option since it will affect the replicating cells in the lesion without affecting the others, even though they were not oncological cells.

Although intratumoral administration of bleomycin is usually recommended in human treatments [17], we decided to use a systemic administration whereas this disease consists of an heterogeneous tissue; thus, the local injection of bleomycin would probably not provide a uniform distribution inside the granuloma in our case.

Our data also confirm that ECT is very useful when the patient's clinical condition impedes a more aggressive treatment. ECT is a cancer treatment modality that has not been used routinely in nononcological diseases [18]. In this case, this modality of treatment achieved excellent results treating eosinophilic granuloma. The patient regained its general condition after a few days, with a rapid overall improvement. The low doses of bleomycin (11 to 30 times lower dose than the one reported as toxic [19]) had no undesired effects. However, the treatment provoked a mild swelling of the gingiva and oral discomfort after the ECT. The situation ceased in less than seven days, controlled with nonsteroidal anti-inflammatory drugs (NSAIDs). It must be noticed that, prior to the ECT treatment, the lesion itself made the food and water intake very difficult. Thus, this side effect was 
quite limited in time and it was actually compensated by the efficacy of the ECT.

The ECT proves to be very useful in cases of a large size granuloma that cannot be reduced considerably by standard treatments based on immunosuppression. The major advantage of ECT could be for treating cases where the owner rejects the radical surgical excision or in those cases where high dose corticosteroids therapy is not a viable option.

\section{Conflict of Interests}

The authors declare that there is no conflict of interests regarding the publication of this paper.

\section{Acknowledgments}

The authors are grateful to Dr. Minatel for the histopathologic processing samples. F. Maglietti and N. Olaiz have fellowships from Consejo Nacional de Investigaciones Cientificas y Tecnicas (CONICET), S. Michinski has a fellowship from Instituto Tecnológico de Buenos Aires (ITBA), and G. Marshall is Member of CONICET. This work is supported by grants from CONICET (PIP 1087/05) and Universidad de Buenos Aires (UBACyT X132/08) and CONICET STAN 599/11.

\section{References}

[1] W. P. Bredal, G. Gunnes, I. Vollset, and T. L. Ulstein, "Oral eosinophilic granuloma in three Cavalier King Charles spaniels," Journal of Small Animal Practice, vol. 37, no. 10, pp. 499-504, 1996.

[2] B. R. Madewell, A. A. Stannard, L. T. Pulley, and V. G. Nelson, "Oral eosinophilic granuloma in Siberian Husky dogs," Journal of the American Veterinary Medical Association, vol. 177, no. 8, pp. 701-703, 1980.

[3] J. M. Sykes IV, M. M. Garner, L. L. Greer et al., "Oral eosinophilic granulomas in tigers (Panthera tigris)-a collection of 16 cases," Journal of Zoo and Wildlife Medicine, vol. 38, no. 2, pp. 300-308, 2007.

[4] M. J. Lommer, "Oral inflammation in small animals," Veterinary Clinics of North America: Small Animal Practice, vol. 43, no. 3, pp. 555-571, 2013.

[5] V. A. Fadok, "Granulomatosis dermatitis in dogs and cats," Seminars in Veterinary Medicine \& Surgery, vol. 2, no. 3, pp. 186194, 1987.

[6] S. J. Key, C. J. O'Brien, K. C. Silvester, and S.-J. Crean, "Eosinophilic granuloma: Resolution of maxillofacial bony lesions following minimal intervention. Report of three cases and a review of the literature," Journal of Cranio-Maxillofacial Surgery, vol. 32, no. 3, pp. 170-175, 2004.

[7] K. A. Potter, R. D. Tucker, and J. L. Carpenter, "Oral eosinophilic granuloma in Siberian huskies," Journal of the American Animal Hospital Association, vol. 16, no. 4, pp. 595-600, 1980.

[8] L. M. Mir, "Bases and rationale of the electrochemotherapy," European Journal of Cancer, Supplement, vol. 4, no. 11, pp. 3844, 2006.

[9] T. Kotnik, P. Kramar, G. Pucihar, D. Miklavčič, and M. Tarek, "Cell membrane electroporation-part 1: the phenomenon," IEEE Electrical Insulation Magazine, vol. 28, no. 5, pp. 14-23, 2012.
[10] M. L. Yarmush, A. Golberg, G. Serša, T. Kotnik, and D. Miklavčič, "Electroporation-based technologies for medicine: principles, applications, and challenges," Annual Review of Biomedical Engineering, vol. 16, pp. 295-320, 2014.

[11] M. Marty, G. Sersa, J. Garbay et al., "Electrochemotherapy—an easy, highly effective and safe treatment of cutaneous and subcutaneous metastases: results of ESOPE (European Standard Operating Procedures of Electrochemotherapy) study," European Journal of Cancer Supplements, vol. 4, pp. 3-13, 2006.

[12] M. Cemazar, Y. Tamzali, G. Sersa et al., "Electrochemotherapy i $\mathrm{n}$ veterinary oncology," Journal of Veterinary Internal Medicine, vol. 22, no. 4, pp. 826-831, 2008.

[13] Local normative law in Argentina, (Law no. 14,072), http:// www.infoleg.gob.ar/infolegInternet/anexos/55000-59999/56727/ norma.htm.

[14] Weight to body surface area conversion, http://www.merckvetmanual.com $/ \mathrm{mvm} / \mathrm{htm} / \mathrm{bc} /$ tref9.htm.

[15] WHO Handbook for Reporting Results of Cancer Treatment, WHO Offset Publications no. 48, World Health Organization, Geneva, Switzerland, 1979.

[16] L. G. Campana, S. Mocellin, M. Basso et al., "Bleomycinbased electrochemotherapy: clinical outcome from a single institution's experience with 52 patients," Annals of Surgical Oncology, vol. 16, no. 1, pp. 191-199, 2009.

[17] B. Mali, T. Jarm, M. Snoj, G. Sersa, and D. Miklavcic, "Antitumor effectiveness of electrochemotherapy: a systematic review and meta-analysis," European Journal of Surgical Oncology, vol. 39, no. 1, pp. 4-16, 2013.

[18] Y. Tamzali, J. Teissie, and M. P. Rols, "Cutaneous tumor treatment by electrochemotherapy: preliminary clinical results in horse sarcoids," Revue de Medecine Veterinaire, vol. 152, no. 8-9, pp. 605-609, 2001.

[19] R. W. Fleischman, J. R. Baker, G. R. Thompson et al., "Bleomycin-induced interstitial pneumonia in dogs," Thorax, vol. 26, no. 6, pp. 675-682, 1971. 

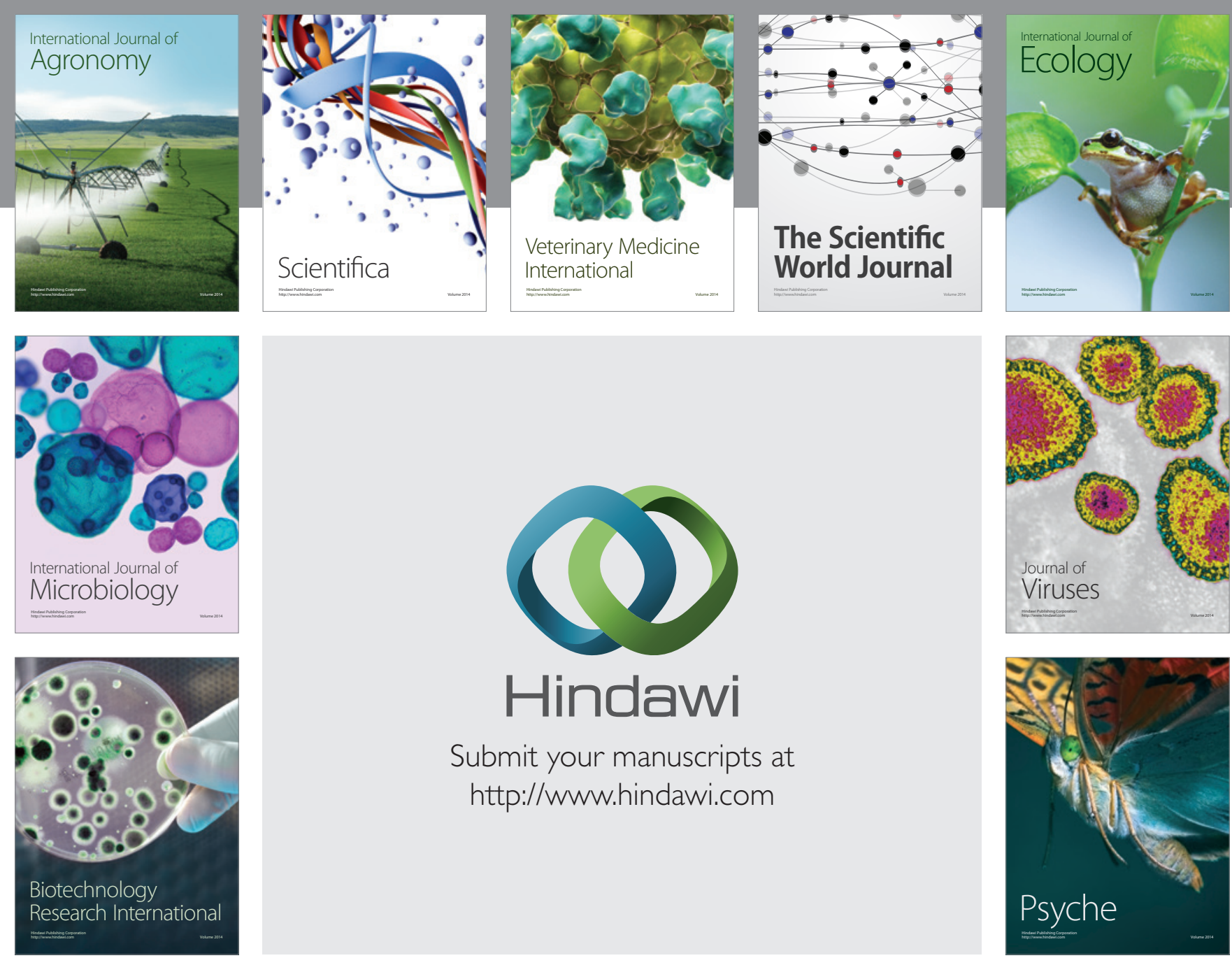

Submit your manuscripts at http://www.hindawi.com
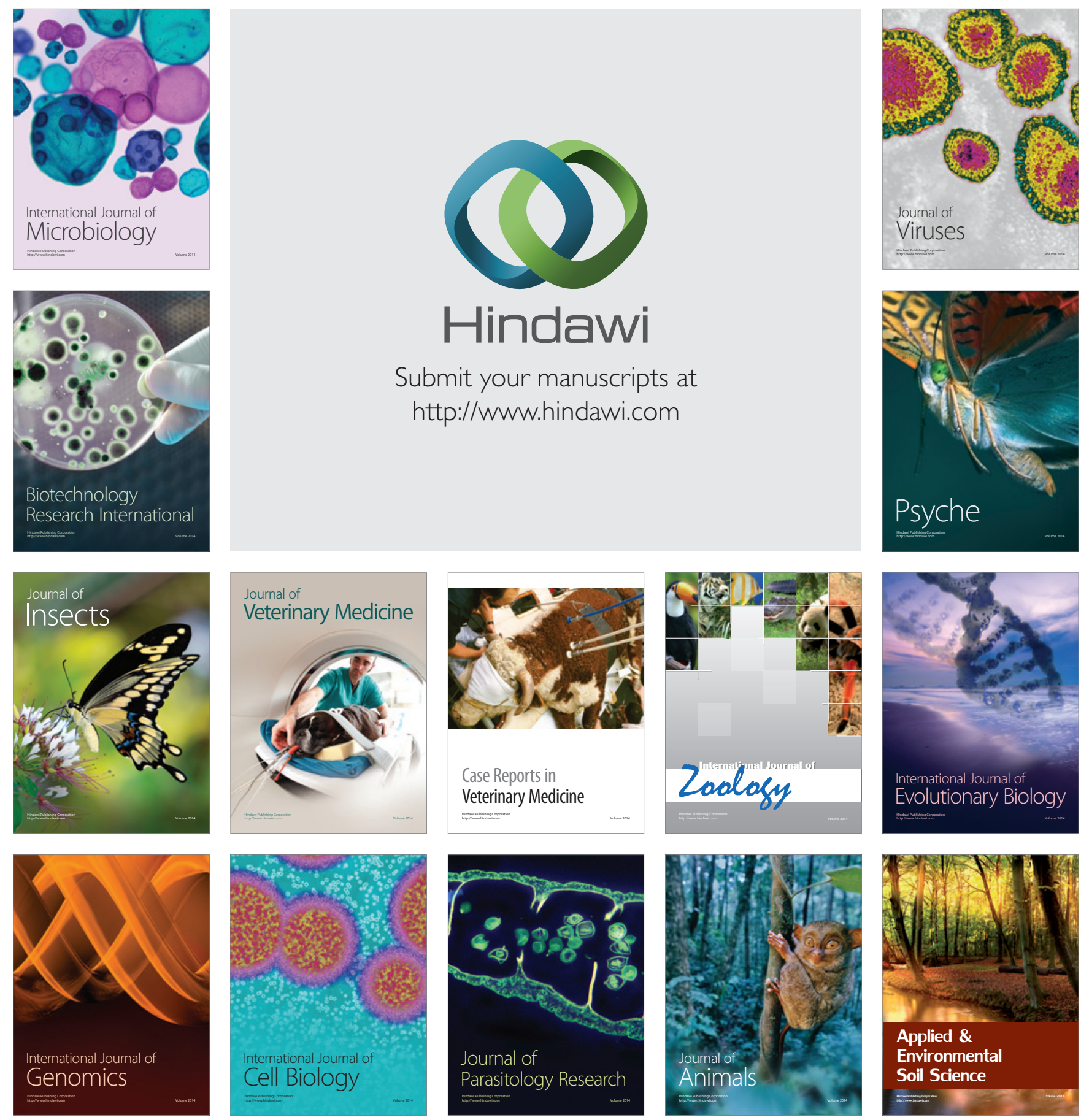